\title{
Vertical-longitudinal coupling induced by wakefields at the Cornell Electron-Positron Storage Ring Test Accelerator
}

\author{
S. T. Wang, ${ }^{*}$ J. D. Perrin, S. Poprocki, W. Hartung, ${ }^{\dagger}$ and D. L. Rubin \\ Cornell Laboratory for Accelerator-based Sciences and Education, Cornell University, \\ Ithaca, New York 14853, USA
}

(Received 15 November 2017; published 5 February 2018)

\begin{abstract}
Transverse vertical wakefields can increase vertical emittance and distort the phase space of a bunch in a storage ring. Here we report recent measurements and simulations of these effects from wakefields from movable scrapers at the Cornell electron-positron storage ring test accelerator. Charge-dependent vertical beam size growth was observed with a single scraper inserted through the top of the storage ring vacuum chamber. No change in the beam size was observed with top and bottom scrapers inserted symmetrically. The apparent growth in the vertical beam size was due in large part to the $y z$ coupling (vertical crabbing) induced by the transverse monopole wake of the asymmetric scraper configuration. We explored this $y z$ coupling and thus the orientation at the observation point by varying the vertical betatron phase advance between the vertical beam size monitor and the scrapers. In addition, we found that existing residual, current-independent $y z$ coupling, perhaps due to nonzero vertical dispersion in the rf cavities, could be compensated by the scraper wake. Predictions from tracking simulations are in good agreement with the measurements. Moreover, the vertical beam size as a function of vertical displacement at narrow-gap chambers (closed scrapers and undulator chamber) was measured. We found the transverse wakefield produced by the off-axis beam could also introduce a $y z$ tilt as well as dilute the beam emittance.
\end{abstract}

DOI: 10.1103/PhysRevAccelBeams.21.024401

\section{INTRODUCTION}

Charged particles moving in vacuum chambers can generate electromagnetic fields called wakefields. If the vacuum chambers are nonuniform or have finite conductivity, or if the bunch is displaced from the axis of the chamber, the wakefields can act back on the particles so as to affect the beam emittance and dynamics [1]. Wakefield effects have been a long-standing topic in accelerator physics, and they have gained more attention in recent years as accelerator projects have sought higher luminosity and higher beam brightness. In a synchrotron light source, transverse wakefields become an important source of emittance dilution with high beam current. Furthermore, insertion devices with narrow vertical gaps are now being used to enhance brightness of x-ray beams [2-4], resulting in stronger transverse wakes. The wakefields can ultimately limit beam brightness via emittance dilution or cause beam instability.

\footnotetext{
*sw565@cornell.edu

Present address: Facility for Rare Isotope Beams, Michigan State University, East Lansing, MI 48824.

Published by the American Physical Society under the terms of the Creative Commons Attribution 4.0 International license. Further distribution of this work must maintain attribution to the author(s) and the published article's title, journal citation, and DOI.
}

The Cornell Electron-positron Storage Ring (CESR) was built in a half-mile circumference tunnel on the Cornell University campus in 1979. The ring stores counterrotating beams of electrons and positrons. Positrons circulate in the clockwise direction in CESR and electrons in the counterclockwise direction as shown in Fig. 1. CESR operated as an $e^{+} e^{-}$high energy physics collider for nearly three decades. Since the conclusion of the colliding beam program in 2008, CESR has been primarily used as a dedicated $\mathrm{x}$-ray source, the Cornell High Energy Synchrotron Source (CHESS). Because the ring energy can be easily configured in the range of 1.5 to $5.3 \mathrm{GeV}$, CESR has also been used as a test accelerator (CESRTA) for exploring the physics and technologies of low emittance rings since 2008 [5].

One aspect of the low emittance studies in CESR was to predict and measure intrabeam scattering (IBS). During an IBS measurement in 2012 [6], an abnormal vertical beam size growth at high current was observed. This growth could not be explained by IBS models, which lead us to investigate wakefield effects. One hypothesis was that wakefields from geometries without up-down symmetry were the source of the vertical beam size growth. In CESR, there are slots for vacuum pumping that produce asymmetric wakes [7]. Our hypothesis led us to develop a method to study asymmetric wakes in a controlled manner using a pair of independently adjustable vertical scrapers. 


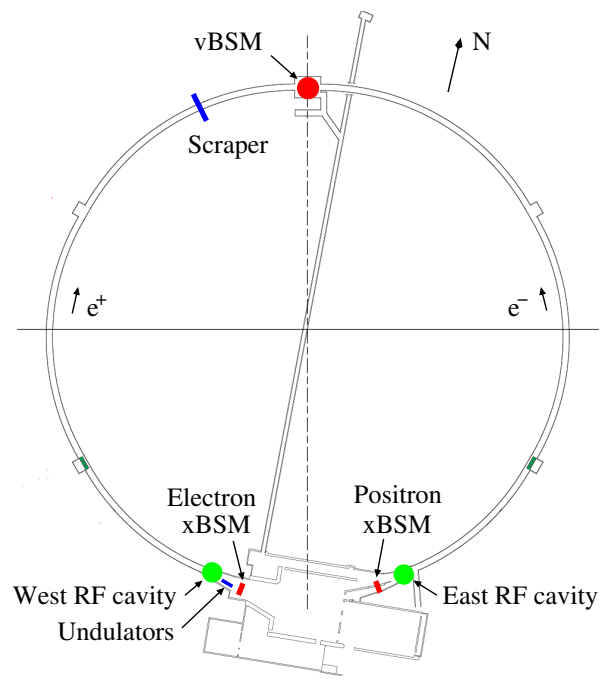

FIG. 1. CESR layout showing the locations of rf cavities, undulators, scrapers, x-ray beam size monitors (xBSM) and visible-light beam size monitor (vBSM).

In 2014, we observed a current-dependent growth in vertical beam size of a single electron bunch at low energy $(2.1 \mathrm{GeV})$ upon inserting a single vertical scraper into the vacuum chamber [7]. No change in the vertical beam size was observed with top and bottom scrapers inserted symmetrically. The growth in beam size is due to the short-range transverse monopole wake associated with the asymmetric scraper configuration [8].

The transverse monopole wake refers to the lowest term $(m=0)$ in an expanded Taylor series of the transverse wakefield $[9,10]$. In a vacuum pipe with up-down symmetry, the transverse monopole wake is zero. The second lowest term, the dipole wake term, is proportional to the offset of the drive particle, but the transverse kick is independent of the witness particle displacement. Additionally, there is a quadrupolar wake term, producing a transverse kick proportional to the offset of the witness particle [9]. The quadrupolar term vanishes in the case of axial symmetry, though the dipole term does not. Note that the quadrupolar term is different from the traditional quadrupole wake $(m=2)$ which is proportional to the square of the drive particle displacement and does not vanish in the axisymmetric case.

In Fall 2014, an undulator chamber with a gap of $4.5 \mathrm{~mm}$ and length of $3.5 \mathrm{~m}$ was installed in CESR to accommodate two undulators for $\mathrm{x}$-ray users. Each undulator is $\sim 1.5 \mathrm{~m}$ long, consisting of 104 poles of permanent magnets [11]. The unique feature of this undulator is that its deflection parameter $K$ can be easily adjusted by adjusting the longitudinal offset between the top and the bottom plates of magnets. However, because of its narrow gap, the undulator chamber could produce a transverse wake which can affect the beam.

In this paper, we present detailed measurements of projected beam sizes as a function of beam current under different lattice conditions and scraper configurations. We calculate the transverse monopole wake of the asymmetric scraper configuration and incorporate it in a tracking simulation. The good agreement between the simulations and measurements shows that the charge-dependent vertical beam size growth can be largely explained by the vertical-longitudinal $(y z)$ coupling induced by the transverse monopole wake. We confirm that the local $y z$ tilt depends on the vertical betatron phase advance with respect to the source of the wake, and that the existing residual $y z$ coupling can be compensated by the scraper wakefield. In addition, the vertical beam size is measured as a function of the vertical displacement of the beam at both the scraper and the narrow-gap undulator chamber.

The paper is organized as follows: in Sec. II, we briefly describe two mechanisms of generating $y z$ beam tilt and show with a simple model how the transverse monopole wake from an asymmetric scraper configuration induces a $y z$ beam tilt. In Sec. III, the design constraints for different lattices used in the experiments are discussed. A coupling/ dispersion knob was created and used in the measurement to induce a current-independent beam tilt in the rf cavity. In $\mathrm{Sec}$ IV, we describe the procedure to acquire the transverse wakefield with numerical simulation tools. The tracking simulation including the numerically calculated transverse monopole wake of the scraper is also discussed. In Sec. V, we discuss the experimental setup and procedure in detail. The current-dependent beam size is measured as a function of the phase advance between scraper and observation point, the scraper configuration, and the vertical dispersion in the rf cavity. The simulation results are then compared with the measurements. In Sec. VI, we present the measured beam size as a function of the vertical displacement of the beam at the scraper and inside the undulator chamber. A discussion and conclusion are provided in the last two sections.

\section{VERTICAL-LONGITUDINAL TILT}

In storage rings, horizontal dispersion in the rf cavity introduces horizontal-longitudinal coupling, contributing to beam tilt in the $x z$ plane [12]. Similarly, vertical dispersion in the rf cavity will induce vertical-longitudinal coupling. This $y z$ coupling can be characterized by a dispersion term $\zeta_{b}$ that appears in the normal mode decomposition of the one-turn transfer matrix [12]. Here we denote the normal-mode coordinates by $(a, b, c)$, in contrast to the lab frame denoted by $(x, y, z)$. The beam tilt angle in the $y z$ plane $\theta_{y z}$ can be written as [12]

$$
\theta_{y z}=\sqrt{\frac{\beta_{b}}{\beta_{c}}} \bar{H}_{35},
$$

where $\beta_{b}$ and $\beta_{c}$ are the vertical and longitudinal Twiss parameters, respectively, and $\bar{H}$ is the coupling matrix 
derived from the one-turn transfer matrix. The contribution to the tilt angle due to the vertical dispersion in the rf cavity is approximately

$$
\theta_{y z} \approx \zeta_{b}-\frac{\alpha_{c}}{\beta_{c}} \eta_{b}
$$

Here $\alpha_{c}$ and $\beta_{c}$ are the Twiss parameters, $\eta_{b}$ is referred to as normal-mode dispersion and $\zeta_{b}$ is the normal-mode crabbing dispersion [12]. The $y z$ tilt induced by the dispersion in the rf cavity does not depend on the beam current.

Another contribution to the tilt angle $\theta_{y z}$ comes from the transverse wakefield. Here we use a simple model to describe the vertical-longitudinal coupling induced by the transverse monopole wake of the asymmetric scraper configuration.

When the bunch travels past the scraper, each particle will experience a vertical kick $\Delta y^{\prime}$ due to the transverse wake $W_{\perp}$ induced by the drive bunch:

$$
\Delta y^{\prime}=W_{\perp} \frac{e q}{E},
$$

where $q$ is the total bunch charge, $e$ is the electron charge, and $E$ is the beam energy. If the transverse wake $W_{\perp}$ is constant, the kick induced by the wake is similar to a dipole error which only distorts the closed orbit without affecting the emittance. However, in general, $W_{\perp}(z)$ is a function of $z$, the particle's longitudinal position relative to the bunch centroid. Hence, the wakefield can produce a $y z$ coupling to the beam. The contribution to the $y z$ tilt $\theta_{y z}$ can be estimated as follows.

If the particle gets a vertical kick $\Delta y^{\prime}$ at location $s_{0}$, the change in the closed orbit $y(s)$ at location $s$ will be

$$
y(s)=\frac{\Delta y^{\prime} \sqrt{\beta_{y}(s) \beta_{y 0}}}{2 \sin \pi Q_{y}} \cos \left[\Delta \phi_{y}(s)-\pi Q_{y}\right],
$$

where $s$ is the location of the particle in the accelerator, $\Delta \phi_{y}(s)$ is the vertical phase advance from $s_{0}$ to $s, Q_{y}$ is the vertical tune, and $\beta_{y}(s)$ and $\beta_{y 0}$ are the vertical betatron functions at $s$ and $s_{0}$, respectively.

In the general case $W_{\perp}(z)$ and hence $\Delta y^{\prime}$ depends on $z$, so a particle at the head of the bunch gets a different vertical kick from a particle at the tail of the bunch. Thus $y(s)$ is different between the head and the tail of the bunch, producing a tilt in the $y z$ plane. To linear order in $z$, we can write $W_{\perp}(z) \approx A z$, in which case the tilt angle $\theta_{y z}$ is given approximately in the limit of vanishing synchrotron oscillations by

$$
\begin{aligned}
\theta_{y z}(s) & \approx \frac{y(s)}{z} \\
& \approx A \frac{e q \sqrt{\beta_{y}(s) \beta_{y 0}}}{2 E \sin \pi Q_{y}} \cos \left[\Delta \phi_{y}(s)-\pi Q_{y}\right] .
\end{aligned}
$$

From Eq. (5), we see the $y z$ tilt due to the wakefield depends on the vertical betatron functions at both the element inducing the wakefield and the observation point, and the vertical betatron phase advance between the wake element and the observation point. As shown in the following sections, although $W_{\perp}(z)$ does not always have a linear relationship with $z$, Eq. (5) reasonably represents the effective $y z$ tilt induced by the wakefield. A more detailed general derivation of the $y z$ tilt due to transverse wakefield using the one-turn matrix can be found in the Appendix.

It is worth noting here that the beam tilts in the $y z$ plane with the "banana-shape" characteristic of a head-tail instability. Unlike the head-tail instability, where the projected vertical beam size oscillates in time at synchrotron frequency, here the beam tilt is fixed with an angle that depends on phase advance from source of the wake. As will be described later, we observe no oscillation in our turn-byturn measurement of projected beam size within 1024 turns ( $\sim 68 T_{s}$, where $T_{s} \approx 38 \mu$ s is the synchrotron oscillation period).

\section{LATTICE AND COUPLING BUMP}

As mentioned in the previous section and evident from Eq. (5), varying the vertical betatron phase advance between the wake element and the observation point will change the beam $y z$ tilt at the observation point. In our experiments, the scraper is the wake element and the $\mathrm{x}$-ray beam size monitor (xBSM) is the observation point. To test this hypothesis, we created eleven positron lattices that differ only in the vertical betatron phase advance between the scraper and the $e^{+} \mathrm{xBSM}\left(\phi_{\mathrm{so}}\right)$. The baseline lattice is labeled as lat ${ }^{+}(0)$, the ring parameters of which are listed in Table I. Ten more sets of optics are created based on lat ${ }^{+}(0)$ by varying the $\phi_{\text {so }}$ with a step of $\Delta \phi=0.314\left(18^{\circ}\right)$. The tunes and the vertical betatron functions at the scraper and XBSM are the same for all the lattices. As an example, the differences in the $\beta_{y}$, the vertical dispersion $\eta_{y}$ and the vertical betatron phase for the two lattices $\operatorname{lat}^{+}(0)$ and $\operatorname{lat}^{+}(-54)$ are shown in Fig. 2. In lattice $\operatorname{lat}^{+}(-54)$, the

TABLE I. CESRTA machine parameters.

\begin{tabular}{lcc}
\hline \hline Beam energy $(\mathrm{GeV})$ & $E$ & 2.085 \\
\hline Circumference $(\mathrm{m})$ & $L$ & 768 \\
Transverse damping time $(\mathrm{ms})$ & $\tau$ & 56.6 \\
Momentum compaction & $\alpha_{p}$ & 0.0068 \\
Nominal rf voltage $(\mathrm{MV})$ & $V_{\mathrm{rf}}$ & 5.8 \\
Synchrotron tune & $Q_{s}$ & 0.067 \\
Horizontal tune & $Q_{x}$ & 14.573 \\
Vertical tune & $Q_{y}$ & 9.629 \\
Horizontal emittance (nm rad) & $\epsilon_{a}$ & $\sim 3$ \\
Vertical emittance (pm rad) & $\epsilon_{b}$ & $10-20$ \\
\hline \hline
\end{tabular}



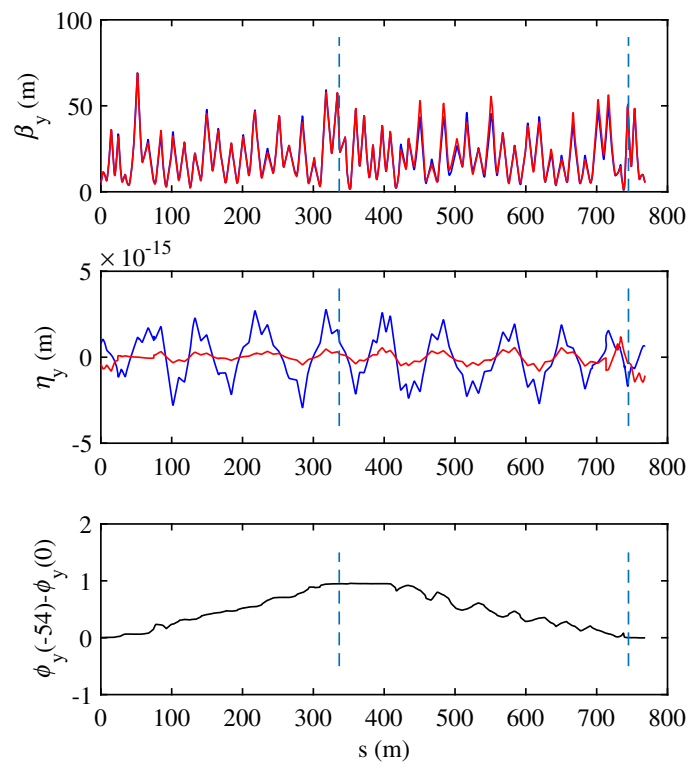

FIG. 2. The vertical Twiss parameter $\beta_{y}$ and the dispersion $\eta_{y}$ of two lattices: $\operatorname{lat}^{+}(0)$ (blue line) and $\operatorname{lat}^{+}(-54)$ (red line). The bottom plot shows the vertical betatron phase difference in units of radians between $\operatorname{lat}^{+}(-54)$ and $\operatorname{lat}^{+}(0)$. Two dashed lines indicate the locations of the scraper $(s=336.5 \mathrm{~m})$ and the $e^{+}$ $\mathrm{xBSM}(s=774.9 \mathrm{~m})$.

phase advance between the scraper and the $e^{+}$xBSM is changed by $-0.942\left(-54^{\circ}\right)$ from lat ${ }^{+}(0)$.

Besides the lattices for positrons, two lattices for electrons, lat ${ }^{-}(0)$ and $\operatorname{lat}^{-}(45)$, were created. Lattices for electrons and positrons are different because the source points for $\mathrm{x}$-ray beam size monitors for electrons and positrons are different. As the name indicates, the phase advance between the scraper and the $e^{-} \mathrm{xBSM}$ in the lat ${ }^{-}(45)$ lattice is $0.785\left(45^{\circ}\right)$ larger than that in the $\operatorname{lat}^{-}(0)$.

As will be discussed in the next section, the transverse monopole wake of the asymmetric scraper configuration is obtained from numerical simulations. After incorporating the wakefield into the lattice and calculating the one-turn matrix, we can compute the beam $y z$ tilt at the observation point using Eq. (1). Figure 3 shows the calculated $y z$ tilt at the $e^{+}$xBSM for the eleven lattices with a bunch current of $4 \mathrm{~mA}$. The evident sinusoidal dependence of tilt angle on phase advance is consistent with Eq. (5). We have also obtained the beam $y z$ tilt from tracking simulations as discussed in the next section. In Fig. 3, a scaling factor is needed for quantitative agreement between the one-turn matrix case and the tracking case; we will return to this discrepancy in Sec. V C. We also find that the wakefield generates vertical dispersion as shown in Fig. 4(a). The vertical dispersion will of course increase the vertical emittance.

If the beam had a $y z$ tilt at the xBSM with both scrapers retracted (no wakefields), the $y z$ tilt induced by the wakefield from inserting the top or bottom scraper could either reduce or increase the beam tilt. In order to study the $y z$ tilt

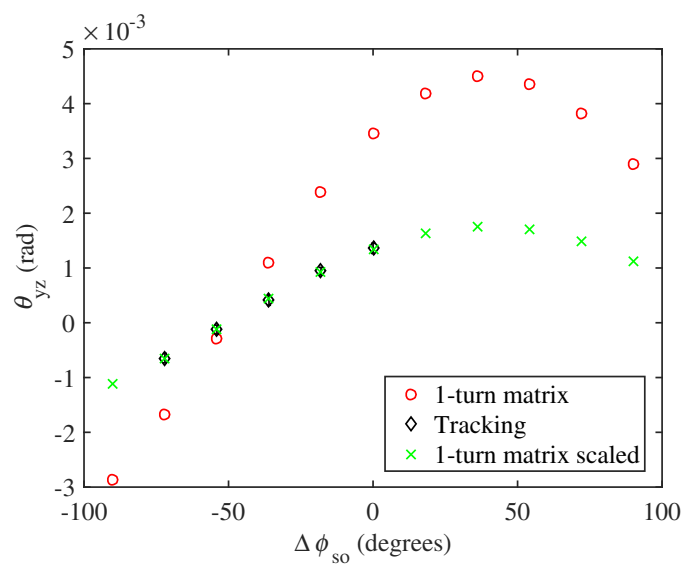

FIG. 3. The $y z$ tilt angle $\theta_{y z}$ at the $e^{+}$xBSM calculated from the one-turn matrix (red circles and green crosses) for 11 lattices, and from tracking simulations (black diamonds) for five lattices. $\Delta \phi_{\text {so }}$ is the change of the vertical betatron phase difference between the scraper and the $\mathrm{xBSM}$ relative to $\operatorname{lat}^{+}(0)$.
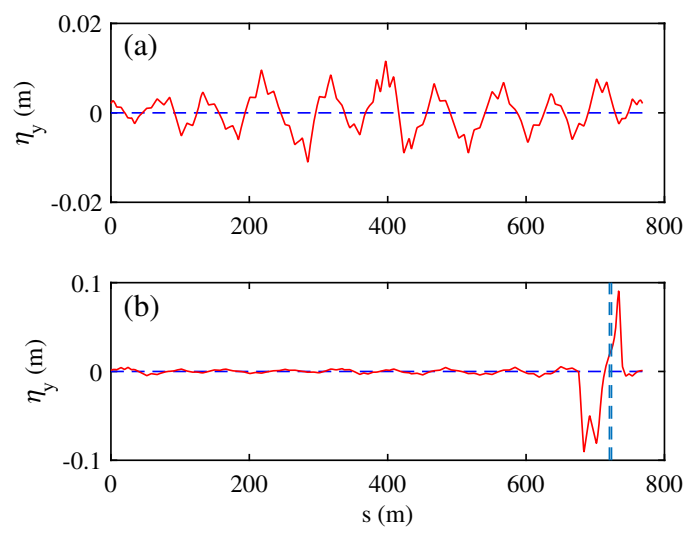

FIG. 4. (a) The vertical dispersion $\eta_{y}$ of the lattice lat $^{-}(0)$ without (blue dashed line) and with (red line) the wake element. (b) $\eta_{y}$ of the lattice lat ${ }^{-}(0)$ with the coupling bump cp11 set at 0 (blue dashed line) and $+10 \mathrm{k}$ (red line). All the $\eta_{y}$ shown are calculated from lattice models. The vertical dashed line indicates the location of the east rf cavity.

effect, we created a closed dispersion/coupling bump cp11 (or cp10) in the east (or west) rf region to generate a finite vertical dispersion $\left(\eta_{y}\right)$ in the east (or west) rf cavity. This vertical dispersion in the rf cavity will then induce a preexisting (current-independent) $y z$ tilt. Using this coupling bump cp11, the preexisting $y z$ tilt at the $e^{-}$xBSM can be varied. Figure 4(b) shows the vertical dispersion before and after setting the coupling bump $(\mathrm{cp} 11=+10 \mathrm{k})$; the $\eta_{y}$ change in the east rf cavity is about $+30 \mathrm{~mm}$.

\section{SIMULATION}

\section{A. Transverse monopole wake}

The ACE3P electromagnetic simulation suite was used to calculate the wakefields [13]. The three-dimensional 
(a)
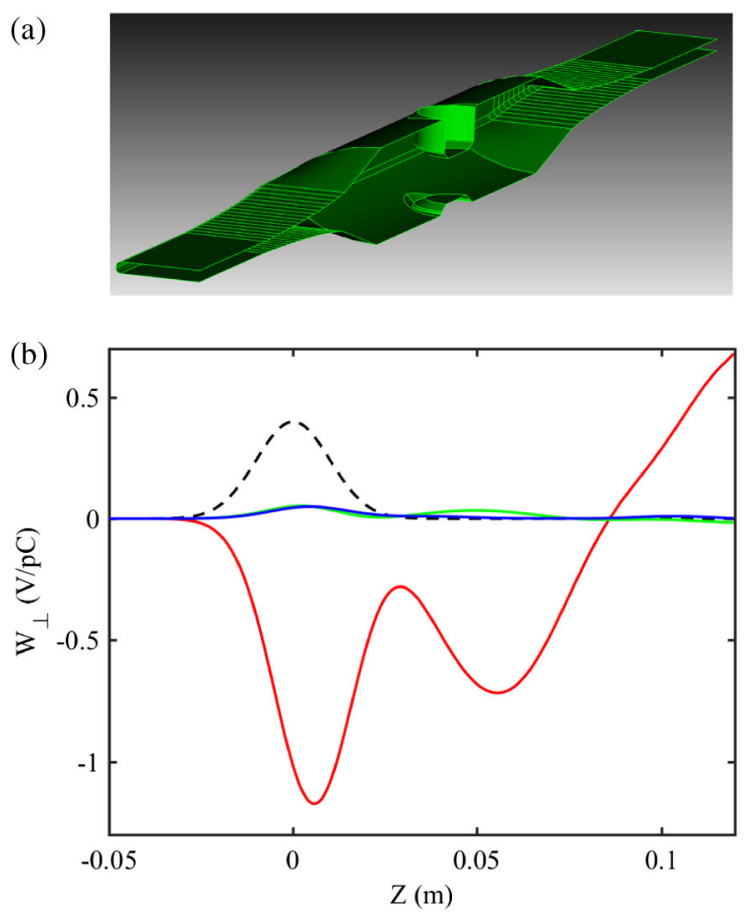

FIG. 5. (a) CUBIT half model of the scrapers, including artificial tapers, with top scraper inserted and bottom scraper retracted. (b) Transverse wakes of the asymmetric scrapers: monopole (red), dipole (green), and quadrupolar (blue) term. The dipole and quadrupolar terms have been multiplied by a factor of $\Delta y=$ $0.5 \mathrm{~mm}$ to compare with the transverse monopole term. Dashed line: bunch distribution $\left(\sigma_{z}=10 \mathrm{~mm}\right)$.

structure of the asymmetric scraper as shown in Fig. 5(a) was constructed using the finite element mesh toolkit CUBIT [14]. This CUBIT model was imported to the time domain wakefield solver T3P to calculate the longitudinal wake. We ran T3P at the National Energy Research Scientific Computing Center [15]. The charged bunch was modeled as a Gaussian distribution with $\sigma_{z}=10 \mathrm{~mm}$ calculated from the lattice model at the experimental synchrotron tune. Longitudinal wakes were computed with the drive bunch at each of three vertical positions $(-\Delta y, 0, \Delta y)$ where $\Delta y=$ $0.5 \mathrm{~mm}$ is the mesh size of the CUBIT model. Using ACE3P [13], the longitudinal wake of the witness particle at a specific vertical position can be found. We did a Taylor expansion of the longitudinal wake in the vertical displacement of the drive bunch and witness particle, and then used the Panofsky-Wenzel theorem [16] to obtain the transverse wake terms from the longitudinal terms. As an example, the transverse monopole, dipole, and quadrupolar wakes of asymmetric scrapers were calculated and shown in Fig. 5(b). It is worth noting here that we used T3P to calculate the short-range wakefield in order to predict the effect of the wake on the particles in the bunch; thus our predictions do not include effects from wakes that endure for multiple turns.

Figure 5(a) shows the half model of the scrapers with top scraper inserted and bottom scraper retracted. The artificial tapers, which match the $7 \mathrm{~mm}$ gap of the closed scrapers, are included in this model in order to satisfy the constraints of the Weiland integration method used in T3P. Wakefields for a separate model of only the artificial tapers were obtained, and subtracted from the wakefields from the scraper model which includes the artificial tapers to yield the wakefields from just the scraper.

As Fig. 5(b) shows clearly, the wakefield depends nonlinearly on particle longitudinal position (z). However, the beam has an effective $y z$ tilt due to the $z$-dependence of the transverse monopole wake. The effective $y z$ tilt factor could be described by $\left.\frac{\partial W_{\perp}(z)}{\partial z}\right|_{z=0}$, the slope of the wake dependence at $z=0$. As indicated in Fig. 5(b), the dipole and quadrupolar wakes were found to be significantly weaker than the transverse monopole wake, so they were not included in the tracking simulation.

\section{B. Tracking simulation}

The particle tracking codes developed for modeling the effect of the wakes make extensive use of BMAD, a subroutine library for relativistic charged-particle dynamics simulations [17]. The scraper was modeled as a wake element with a custom tracking routine, which applies the calculated vertical kick to each macroparticle based on its $z$ position. The bunch was modeled as a distribution of 1000 macroparticles and tracked through a CESRTA lattice for $100 \mathrm{k}$ turns (about five radiation damping times). At every turn the particles' six-dimensional coordinates $\left(x, x^{\prime}, y, y^{\prime}\right.$, $\left.z, z^{\prime}\right)$ at the XBSM location were recorded to construct the sigma matrix, from which the $y z$ tilt and projected $\sigma_{y}$ were calculated [18]. Quantum excitation and damping (stochastic emission of photons) was included. Normally after $50 \mathrm{k}$ turns, the beam reaches equilibrium. The $\sigma_{y}$ averaged over the last $30 \mathrm{k}$ turns is reported as the simulated beam size. The betatron tunes in the simulation are set to match the measured values. The zero current vertical emittance and the pretilt angle at the xBSM source point with zero wakefield are free parameters varied so that simulation matches data.

The real machine has misaligned magnets which lead to lattice errors (beta functions, coupling, vertical emittance, etc.). Correctors such as vertical and horizontal steerings and skew-quadrupoles distributed around the ring are used to compensate the misalignments. After lattice correction and low emittance tuning [19], the ring will in general have a small but nonzero vertical emittance due to residual misalignment of magnets. Since it is not realistic to reproduce the exact machine conditions for simulation, one way to simulate the machine conditions is to generate a lattice which includes misalignment of the magnets and has the desired vertical emittance. The procedure to create such a lattice is described in Ref. [19]. However, as will be mentioned in Sec. V, the pretilt angle at the observation point is another important parameter. It is time consuming 
and impractical to generate a misaligned lattice which meets the requirements for both the vertical emittance and the pretilt angle at the xBSM.

An alternative approach to achieve the desired vertical emittance $\epsilon_{y}$ is to apply a random vertical kick $\Delta y_{e}^{\prime}$ to each particle on every turn. The vertical kick $\Delta y_{e}^{\prime}$ is determined according to

$$
\Delta y_{e}^{\prime}=\sigma_{p_{y}} R=2 R \sqrt{\frac{\Delta \epsilon_{y} \tau_{y}}{\beta_{y_{0}}}},
$$

where $R$ is a random number generated for each particle from a normal distribution, $\tau_{y}$ is the vertical damping time, and $\beta_{y_{0}}$ is the vertical beta value at the lattice's first element where the vertical kick is applied. The $\Delta \epsilon_{y}$ is the emittance growth due to the random kick, which is chosen to match the simulated vertical beam size to the measured vertical beam size at low current $(\sim 1 \mathrm{~mA})$ with both scrapers retracted. The pretilt angle $\theta_{p}$ at the xBSM can be generated by using the coupling knobs (cp10 and cp11) described in Sec. III. The pretilt angle $\theta_{p}$ is determined mainly by matching the simulated $\sigma_{y}$ to the measured data with only one of the scrapers inserted.

\section{EXPERIMENTS}

\section{A. Procedure}

The measurements were done with a single bunch of electrons or positrons stored, in contrast to typical CHESS operations with multiple bunches of electrons and positrons. The measurements have been taken for both positrons and electrons at $2.1 \mathrm{GeV}$ in specie-dependent lattices with common global parameters. The nominal parameters of these lattices are listed in Table I. A tuning procedure which corrects the lattice betatron functions, $x y$ coupling, and vertical dispersion was initially applied to achieve low vertical emittance $\left(\epsilon_{y} \approx 15 \mathrm{pm}\right)$ in the accelerator [19]. During measurements, the bunch dimensions $\left(\sigma_{x}, \sigma_{y}, \sigma_{z}\right)$ were recorded concurrently as the beam current decayed from 4 to $1 \mathrm{~mA}$. Since there is a current-dependent tune shift, in order to avoid a resonance effect on the beam sizes through the entire current range, the ring fractional tunes were carefully set at $Q_{x}=0.573, Q_{y}=0.629, Q_{z}=0.067$ for electrons and $Q_{x}=0.574, Q_{y}=0.620, Q_{z}=0.058$ for positrons at low current $(\sim 1 \mathrm{~mA})$. The measurements for positrons and electrons were taken during different experimental shifts and the machine tunes were set differently.

As the current decayed, turn-by-turn beam positions at 100 beam position monitors (BPMs) were also recorded for 8192 turns at three different currents $\sim 3.5, \sim 2.5$, and $\sim 1.5 \mathrm{~mA}$. Analysis of these turn-by-turn BPM data confirms the current-dependent tune shift and also provides useful information about the closed orbit at different currents. Occasionally at a different current, tunes from a spectrum analyzer were recorded with top scraper inserted or retracted.

The $\sigma_{y}$ of an electron (or positron) bunch was measured by the $e^{-}$(or $e^{+}$) xBSM [20], located in the south of the ring as shown in Fig. 1. This instrument is capable of measuring bunch-by-bunch and turn-by-turn vertical beam size. The $\sigma_{x}$ was monitored by the visible-light beam size monitor (vBSM) utilizing the interferometer method [21]. The $\sigma_{z}$ was measured by streak camera during some experimental runs. However, because of potential instrumental error (noncalibrated), the $\sigma_{z}$ data are not included in this paper. The instruments using visible light are located in the north region of CESR.

The vertical scrapers were also located in the north region of the ring as shown Fig. 1. The gap between top and bottom scrapers is 7 or $50 \mathrm{~mm}$, respectively, when both scrapers are fully inserted or retracted. Before measurements, the beam was carefully centered between the two fully inserted scrapers. The current decay data were collected with both scrapers retracted or with only one of the scrapers fully inserted. The beam size data with both scrapers fully inserted were also taken occasionally, and are very similar to the data measured with both scrapers retracted. This demonstrates that the symmetric scraper configuration did not generate a transverse monopole wake responsible for the vertical beam size blowup.

\section{B. Beam size versus current}

Figure 6 shows the measured beam sizes as a function of the beam current with top scraper inserted (in-out) or retracted (out-out) for the $e^{-}$lattice $\operatorname{lat}^{-}(0)$. With both scrapers retracted, $\sigma_{x}$ increased with current from 1 to $4 \mathrm{~mA}$ while $\sigma_{y}$ remained constant $(\sim 21 \mu \mathrm{m})$. The observed current dependence of horizontal beam size is consistent with IBS [6]. The calculated horizontal beam size growth due to IBS based on Bjorken-Mtingwa formalism with a tail cut is plotted in Fig. 6(a). Since both vertical dispersion and $y z$ coupling were small after low emittance tuning, the effect of IBS is most pronounced in the horizontal plane [6]. Thus, no apparent $\sigma_{y}$ increase was observed due to IBS.

When the top scraper was inserted, the measured $\sigma_{y}$ increased dramatically with the beam current [Fig. 6(b), black crosses]. From analyzing the xBSM data, we find the turn-by-turn beam size is quite stable without showing any sign of the head-tail instability. We attribute the current dependence of vertical beam size growth to the transverse monopole wake generated by the asymmetric scrapers. As Sec. IV shows, the asymmetric scraper configuration (inserted top scraper only) generates a transverse monopole wake, which induces an effective beam tilt $\theta_{y z}$ determined by Eq. (5).

The effect of the $y z$ tilt will be to increase the projected (or measured) vertical beam size $\sigma_{y}$ according to

$$
\sigma_{y}^{2}=\sigma_{y n}^{2}+\left(\sigma_{z} \theta_{y z}\right)^{2},
$$



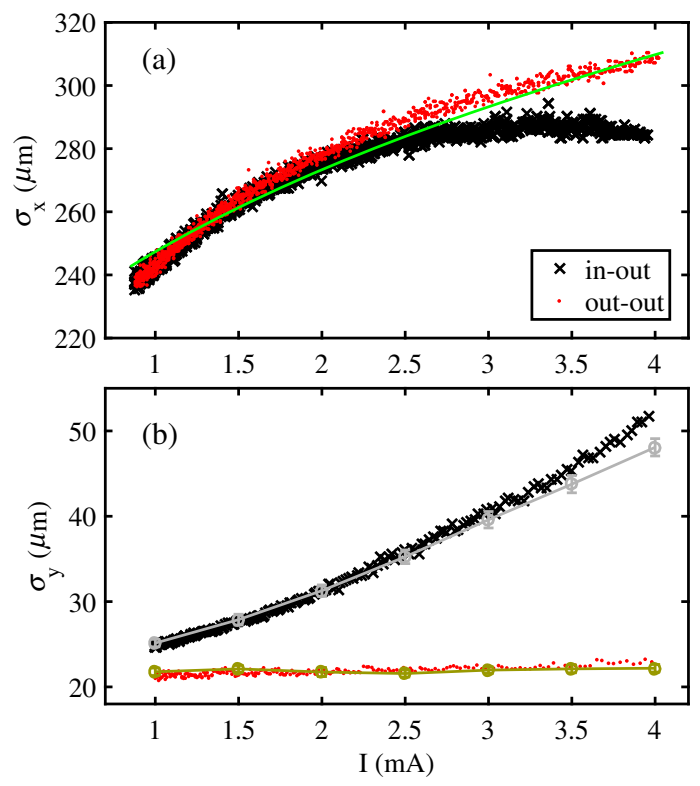

FIG. 6. Beam sizes as a function of the beam current for the $e^{-}$ lattice lat $^{-}(0)$. The red dots are the data measured with both scrapers retracted and the black crosses are the data with top scraper fully inserted. Circles are from tracking simulations. The green line is calculated beam size growth due to IBS with both scrapers retracted.

where $\sigma_{y n}$ is the normal mode vertical beam size. As Eq. (5) indicates, the $y z$ tilt is proportional to the bunch charge $q$. The higher the bunch charge, the larger the tilt. This quantitatively explains the observed current-dependent $\sigma_{y}$ growth. In addition, as Fig. 6(b) shows, the good agreement between the $\sigma_{y}$ from tracking simulation (open circles) and the data shows that the transverse monopole wake produced with the top scraper can explain the vertical beam size growth. The two free parameters for the tracking simulation are the zero current vertical emittance $\epsilon_{y}=11.3 \mathrm{pm}$ and the pretilt angle at the $e^{-} \mathrm{xBSM}, \theta_{p}=-0.3 \mathrm{mrad}$.

An alternative explanation for the current-dependent $\sigma_{y}$ growth might be that it is due to a drift in the orbit with current, which could introduce $x y$ coupling or dispersion. Turn-by-turn BPM data were obtained and closed orbits were extracted at different currents with top scraper inserted or retracted. We found no strong current-dependent orbit shifts with or without the scraper wake. For both cases, the measured difference between the closed orbit at the high current $(3.5 \mathrm{~mA})$ and low current $(1.5 \mathrm{~mA})$ was less than $300 \mu \mathrm{m}$ with an $\mathrm{rms}$ of $\sim 150 \mu \mathrm{m}$. The orbit difference between the cases with top scraper inserted or retracted at the same current is less than $50 \mu \mathrm{m}$ with an rms of $\sim 20 \mu \mathrm{m}$. Therefore, the orbit shift from the scraper wake is negligible, which excludes the possibility that current-dependent orbit drift produces the growth in beam size. In addition, by comparing the tunes with top scraper inserted and retracted at the same current, we found the tune shift due to insertion of the scraper is small $(<0.5 \mathrm{kHz})$ through the entire current range. The ring tunes at low current were carefully chosen to avoid resonant lines in the tune plane. It is very unlikely the vertical beam size growth shown in Fig. 6(b) is due to the current-dependent tune shift. Moreover, the $\sigma_{y}$ increase due to hitting a resonant line would be more abrupt as will be shown in Fig. 11(a). However, the currentdependent tune shift could drag the machine tune closer to a resonant line at large beam current and then the measured vertical beam size increases slightly so that the simulated data deviate more from the measured as shown in Fig. 6(b).

\section{Phase difference}

As Eq. (5) shows, the beam tilt also depends on the vertical betatron phase advance $\Delta \phi_{y}(s)$ between the wake element (scraper) and the observation point (xBSM). Varying $\Delta \phi_{y}(s)$ will change the beam tilt and the projected beam size at the observation point. Therefore, a new $e^{-}$ lattice $\operatorname{lat}^{-}(45)$ was loaded into the accelerator, which differs from the lat $^{-}(0)$ lattice only in the increase in vertical phase advance between scraper and $e^{-}$xBSM by $\pi / 4$. The measured beam sizes in this new lattice are shown in Fig. 7(a).

For the lat ${ }^{-}(45)$ lattice, the $\sigma_{y}$ growth with top scraper inserted is much less than that in lat ${ }^{-}(0)$ (Fig. 6), indicating the dependence of the local tilt on the phase advance with respect to the source of the wake. Indeed the $y z$ tilts calculated from tracking simulation for both lattices shown in Fig. 7(b) confirm a weaker current-dependent tilt in the lat $^{-}(45)$ lattice than that in the lat ${ }^{-}(0)$ lattice.
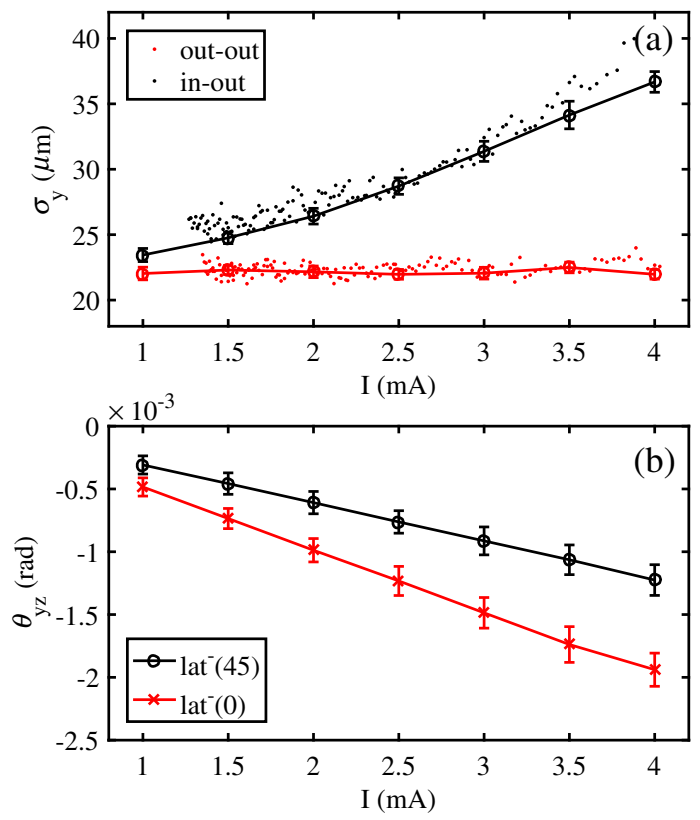

FIG. 7. (a) Measured vertical beam size $\sigma_{y}$ as a function of bunch current for $\operatorname{lat}^{-}(45)$ of electrons (circles: simulation). (b) Tilt angle $\theta_{y z}$ from tracking simulations for $\operatorname{lat}^{-}(0)$ and lat $^{-}(45)$ with top scraper inserted ("in-out"). 

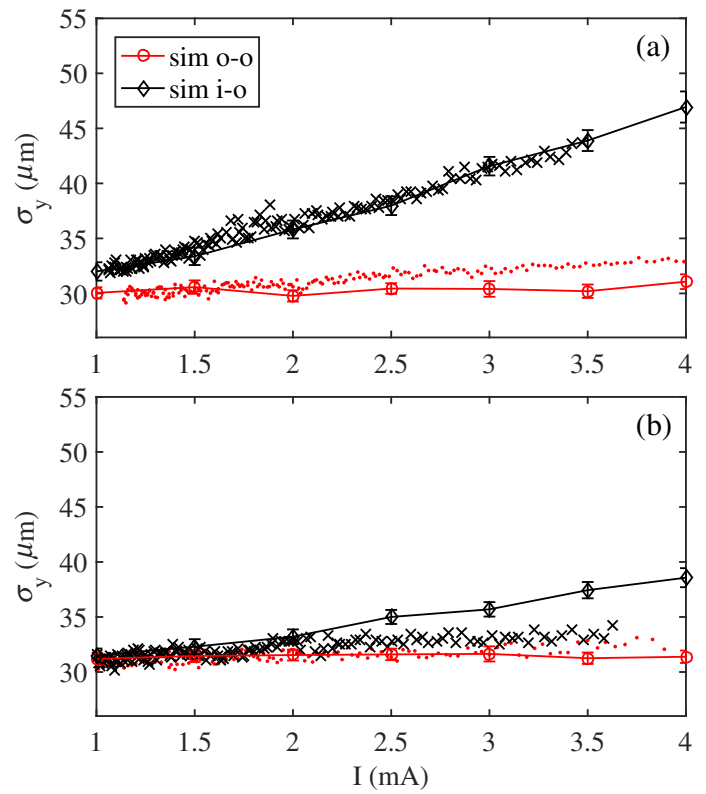

FIG. 8. The measured vertical beam size $\sigma_{y}$ as a function of bunch current with top scraper inserted (black crosses) or retracted (red dots) for two $e^{+}$lattices: (a) $\operatorname{lat}^{+}(0)$ and (b) $l a t^{+}(-54)$. The simulated results are the diamonds and circles.

Similar measurements were also taken for five out of eleven $e^{+}$lattices described in Sec. III. Figure 8 shows the current decay data for two lattices, $\operatorname{lat}^{+}(0)$ and lat $^{+}(-54)$. The vertical beam sizes measured in $\operatorname{lat}^{+}(-54)$ were nearly independent of current when the top scraper was inserted, indicating that the scraper wakefield induces almost zero $y z$ tilt at the $e^{+} \mathrm{xBSM}$ in $\operatorname{lat}^{+}(-54)$. This observation is consistent with the prediction from the one-turn matrix calculation showing the minimum tilt observed in $\mathrm{lat}^{+}(-54)$ (Fig. 3). It also agrees with the simple model described by Eq. (5). The vertical betatron phase advance from the scraper to the $e^{+} \mathrm{xBSM}$ in $\operatorname{lat}^{+}(-54)$ is found to be $10.1 \pi$. With $Q_{y}=9.620$, the calculated cosine term in Eq. (5) is 0.06 , which is near zero and the minimum among all the eleven lattices. As shown in Fig. 8(b), the simulated data deviate significantly from the measured data with top scraper inserted. We will discuss this discrepancy in detail in Sec. VII.

The $y z$ tilt angle of the five lattices at $4 \mathrm{~mA}$ obtained from tracking simulation shows a similar trend as the calculation from the one-turn matrix but with a discrepancy as indicated in Fig. 3. This discrepancy between the tracking simulation and one-turn matrix can be resolved by scaling the $\theta_{y z}$ from one-turn matrix with a factor of 0.4 . The reason for the discrepancy may be due to the fact that the tracking simulation calculates the effective tilt angle of the non-Gaussian profile while the one-turn matrix calculation does not.

\section{Preexisting tilt}

If the beam has a $y z$ tilt at the xBSM with both scrapers out, the $y z$ tilt induced by the wakefield from inserting the
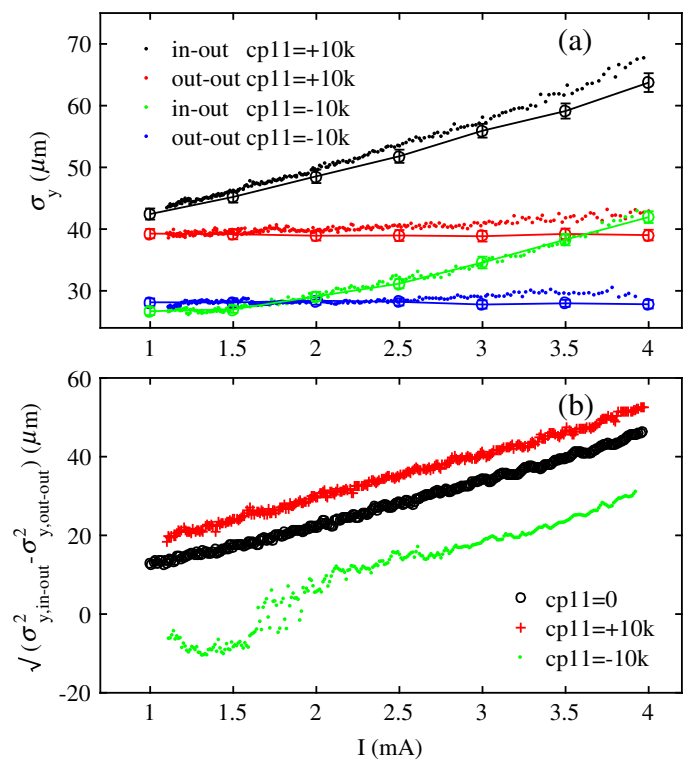

FIG. 9. (a) Measured vertical beam size $\sigma_{y}$ as a function of bunch current with coupling bumps (cp11 = $\pm 10 \mathrm{k})$ in lat $^{-}(0)$ of electrons. (b) Measured vertical beam size contribution due to asymmetric scraper $\left(\sqrt{\sigma_{y, \text { in-out }}^{2}-\sigma_{y, \text { out-out }}^{2}}\right)$ as a function of bunch current.

top scraper can either reduce or increase the beam tilt. Since vertical dispersion in the rf cavity could induce a $y z$ coupling [12], we created a closed dispersion/coupling bump in the east rf region to adjust the vertical dispersion $\left(\left|\eta_{y}\right|\right)$ in the east rf cavity as described in Sec. III. Using this coupling bump, cp11, the current-independent $y z$ tilt at the xBSM can be varied. Measurement data for two cases, $\mathrm{cp} 11=+10 \mathrm{k}\left(\eta_{y}=+30 \mathrm{~mm}\right)$ and $\mathrm{cp} 11=-10 \mathrm{k}$ $\left(\eta_{y}=-30 \mathrm{~mm}\right)$, are shown in Fig. 9(a). For the case $\mathrm{cp} 11=+10 \mathrm{k}$ without top scraper inserted ("out-out"), the $\sigma_{y}$ are much larger than the out-out case with cp $11=0$, which is due to increased vertical emittance after setting $\mathrm{cp} 11=+10 \mathrm{k}$. When the top scraper is inserted (in-out) with cp11 $=+10 \mathrm{k}$, the $\sigma_{y}$ growth due to the wakefield is much larger than that with $\mathrm{cp} 11=0$ through the entire current range as is more clearly seen in Fig. 9(b). On the contrary, for cp11 $=-10 \mathrm{k}$, the $\sigma_{y}$ growth due to the wakefield of the top scraper is less than those with $\operatorname{cp} 11=0$ and $\operatorname{cp} 11=+10 \mathrm{k}$ at higher bunch currents. When the current is between 1 and $1.75 \mathrm{~mA}$, the wakefield even reduces the $\sigma_{y}$. These features are explained below.

Figure 10 shows the equilibrium $y z$ profiles of 1000 particles after tracking $100 \mathrm{k}$ turns with coupling bump set at three different cases: $0,+10 \mathrm{k}$, and $-10 \mathrm{k}$. It directly demonstrates how the beam tilt affects the measured projected $\sigma_{y}$.

With $\eta_{y}=+30 \mathrm{~mm}$ in the east $\mathrm{rf}$ cavity, the $y z$ tilt generated by the dispersion has the same sign (-) as the tilt induced by the wakefield as described in the cartoon of Fig. 10(b). Thus, the scraper wakefield tilted the beam more 


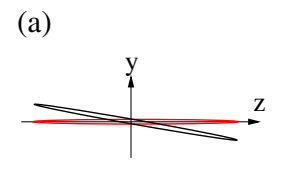

(b)
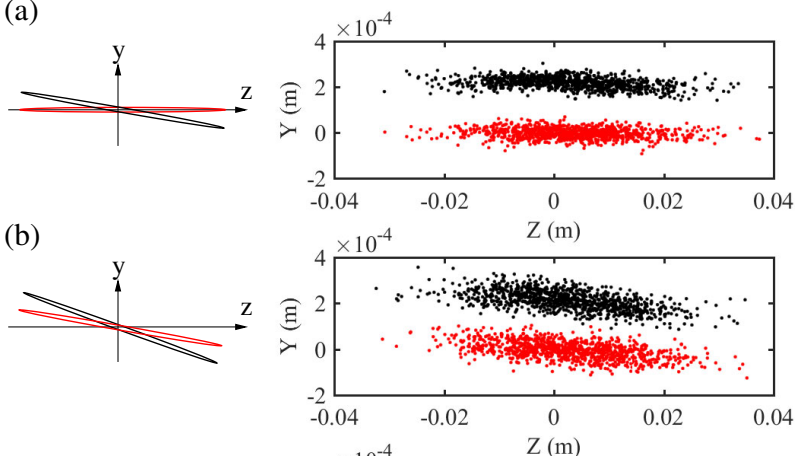

(c)

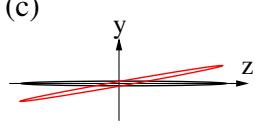

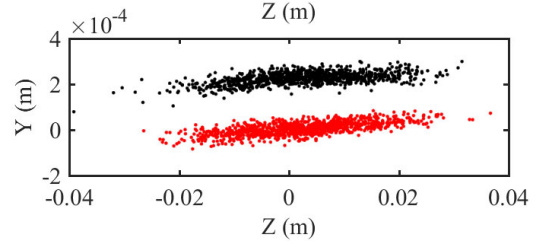

FIG. 10. Right: Particle distributions in the $y z$ plane from simulations for the $e^{-}$lattice lat ${ }^{-}(0)$. Left: Cartoons showing the beam tilt in the $y z$ plane. Red: top scraper retracted. Black: Top scraper inserted. The coupling bump is set to (a) $\mathrm{cp} 11=0$, (b) $\mathrm{cp} 11=+10 \mathrm{k}$, and (c) cp11 $=-10 \mathrm{k}$ with a bunch current of $1 \mathrm{~mA}$.

so that the increase of $\sigma_{y}$ due to the wakefield $\left(\Delta \sigma_{y}=\sqrt{\sigma_{y, \text { in-out }}^{2}-\sigma_{y, \text { out-out }}^{2}}\right)$ is larger than that without pretilt $(\mathrm{cp} 11=0)$ as shown in Fig. 9(b). With $\eta_{y}=$ $-30 \mathrm{~mm}$ in the east rf cavity, the pretilt has opposite sign $(+)$ as the tilt induced by the scraper wakefield as displayed in the cartoon of Fig. 10(c). Thus, the pretilt from dispersion was compensated by the tilt induced by the wakefield so that $\Delta \sigma_{y}$ is less than that without pretilt. Between 1 and $1.75 \mathrm{~mA}$, due to the tilt compensation by the wakefield, the effective beam tilt is less than the pretilt so that $\sigma_{y}$ is smaller with the top scraper inserted than without scrapers. The imaginary $\Delta \sigma_{y}$ are plotted as negative values in Fig. 9(b) to indicate the sign change of $\theta_{y z}$ around $1.75 \mathrm{~mA}$. These measurements demonstrate that beam tilt is the dominant effect induced by the transverse monopole wake from the asymmetric scrapers.

In the above experiments, we collected the data with only the top scraper inserted or with both scrapers retracted. Instead, if only the bottom scraper were inserted, by symmetry the induced wakefields of the bottom scraper will differ from that of the top scraper only by a minus sign, and so will the induced $y z$ tilt at the xBSM. Figure 11(a) plots the measured $\sigma_{y}$ as a function of current in the $e^{-}$ lattice $\operatorname{lat}^{-}(0)$ with $\mathrm{cp} 11=0$ for three different cases: without inserting scrapers $\left(\sigma_{y, \mathrm{oo}}\right)$, with only top scraper inserted $\left(\sigma_{y, \text { io }}\right)$, and with only bottom scraper inserted $\left(\sigma_{y, \mathrm{oi}}\right)$. At $0.6 \mathrm{~mA}, \sigma_{y, \mathrm{oi}}<\sigma_{y, \mathrm{oo}}$ while $\sigma_{y, \text { io }}>\sigma_{y, \text { oo }}$. This is easily explained by the cartoon shown in Fig. 11(a). As discussed above, the wakefield-induced tilt by the top scraper is negative. By symmetry the wakefield-induced
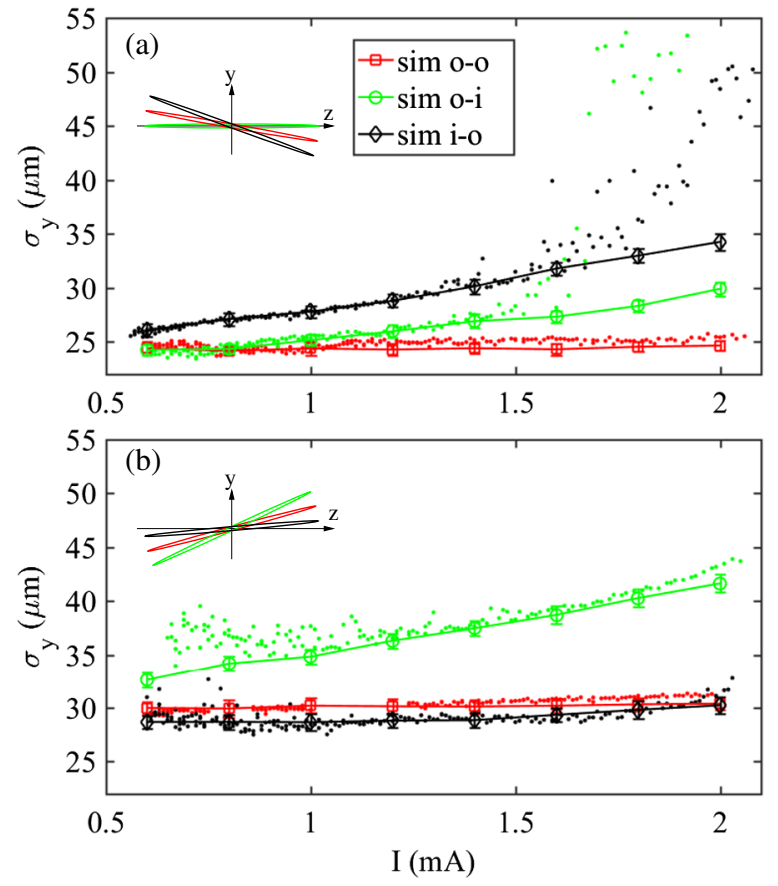

FIG. 11. Measured vertical beam size $\sigma_{y}$ versus current for the $e^{-}$lattice lat ${ }^{-}(0)$ with the coupling bump set at (a) cp11 $=0$ and (b) cp $11=-10 \mathrm{k}$. Small dots: data. Larger symbols with error bars: simulation. Red: both scrapers out (o-o). Black: top scraper inserted (i-o). Green: bottom scraper inserted (o-i). The cartoons indicate the beam tilts at $0.6 \mathrm{~mA}$.

tilt by the bottom scraper is positive. Therefore, there must exist a negative pretilt at the XBSM so that inserting the top scraper tilts the beam more negatively while inserting bottom scraper reduces the beam tilt. The increase or decrease of the $y z$ beam tilt leads to the increase or decrease of the measured $\sigma_{y}$. Since the tilt produced by the wakefield increases with current, at about $0.75 \mathrm{~mA}$, the tilt produced by the bottom scraper overcompensates the pretilt so that $\sigma_{y, \text { oi }}$ starts to become greater than $\sigma_{y, 0 o}$. The beam size measurements between 1.5 and $2 \mathrm{~mA}$ with one scraper inserted are largely scattered. This may be because the machine vertical tune shifted closer to a resonant line when one of the scrapers was inserted.

After setting the coupling bump cp11 $=-10 \mathrm{k}$, the pretilt angle at the xBSM became positive. Now inserting the top scraper reduces the beam $y z$ tilt while inserting the bottom scraper increases the beam $y z$ tilt. Thus, $\sigma_{y, \text { oi }}>$ $\sigma_{y, \text { oo }}$ while $\sigma_{y, \text { io }}<\sigma_{y, \text { oo }}$ as shown in Fig. 11(b). At $\sim 2 \mathrm{~mA}$, we can see $\sigma_{y \text {,io }}$ starts to become greater than $\sigma_{y, \text { oo }}$, indicating the tilt produced by the top scraper has overcompensated the pretilt.

The simulated $\sigma_{y}$ from tracking simulations match the data very well as shown in Figs. 9 and 11. As a summary, Table II lists the simulation parameters: the zero current vertical normal emittance, and the pretilt angle at the xBSM for the simulations shown in these two figures as well as in other figures. As we can see from Table II, the pretilt angle 
TABLE II. Simulation parameters.

\begin{tabular}{lcccc}
\hline \hline \multicolumn{1}{c}{ Lattice } & $\mathrm{cp} 11$ & $\epsilon_{b}(\mathrm{pm} \cdot \mathrm{rad})$ & $\theta_{p}(\mathrm{mrad})$ & Figure \\
\hline$e^{-}$lat $^{-}(0)$ & 0 & 11.3 & -0.3 & 6 \\
$e^{-}$lat $^{-}(45)$ & 0 & 12.0 & 0.0 & 7 \\
$e^{-}$lat $^{-}(0)$ & $+10 \mathrm{k}$ & 28.0 & -1.86 & $9(\mathrm{a})$ \\
$e^{-} \mathrm{lat}^{-}(0)$ & $-10 \mathrm{k}$ & 10.0 & 1.86 & $9(\mathrm{a})$ \\
$e^{-} \mathrm{lat}^{-}(0)$ & 0 & 13.3 & -0.66 & $11(\mathrm{a})$ \\
$e^{-} \mathrm{lat}^{-}(0)$ & $-10 \mathrm{k}$ & 11.2 & 2.02 & $11(\mathrm{~b})$ \\
$e^{+} \mathrm{lat}^{+}(0)$ & 0 & 21.0 & 0.3 & $8(\mathrm{a})$ \\
$e^{+} \mathrm{lat}^{+}(-54)$ & 0 & 23.5 & 0.15 & $8(\mathrm{~b})$ \\
\hline \hline
\end{tabular}

is required to fit most of the data. Although the low emittance tuning corrects the vertical dispersion around the ring, residual vertical dispersion in the rf cavity can introduce a small $y z$ tilt $\theta_{p}$ at the xBSM. Thus, when calculating the vertical emittance of the beam from the measured $\sigma_{y}$, one should take this beam tilt into account. Measurements of projected beam size are incapable of discerning beam tilt directly. However, we find that the scraper can serve as a beam tilt detector by measuring the dependence of projected size on the scraper configuration.

\section{BEAM SIZE VERSUS DISPLACEMENT}

In the above sections, we showed that the wakefield generated by an asymmetric beam pipe can induce a beam $y z$ tilt. Similarly, when a beam passes through a symmetric chamber with a vertical displacement from the chamber axis, the beam will experience a transverse wakefield. In the up-down symmetric case, the transverse monopole term is zero, but the dipole and quadrupolar terms are nonzero and can produce a transverse kick when the beam is displaced. The wakefield can tilt the bunch so as to increase the measured $\sigma_{y}$. Snuverink et al. [22] have demonstrated a charge-dependent beam size growth at the ATF2 beam line using a movable chamber.

Here we measured the vertical beam size when the $y$ position of the beam was varied at the scrapers while both scrapers were fully inserted. The $y$ position of the beam was adjusted using a closed-orbit bump. Five sextupoles at the bump location were turned off to eliminate nonlinear coupling from the sextupoles. The origin $(\Delta y=0)$ is inferred from the bump settings at which beam loss was observed (via a decrease in the stored beam lifetime). The measurements were done with a single electron bunch at three currents: 1,3 , and $4 \mathrm{~mA}$.

As shown in Fig. 12(a), the measured vertical beam size is asymmetric between upward and downward displacement for every case. The minimum $\sigma_{y}$ is at positive $\Delta y$. In addition, the $\Delta y_{\min }$, at which $\sigma_{y}$ reaches the minimum, seems to be dependent on the bunch current. The higher the bunch current, the smaller the $\Delta y_{\min }$. This suggests that there is a preexisting $y z$ tilt at the $e^{-} \mathrm{xBSM}$ when $\Delta y=0$. We know the wakefield increases with the displacement. When the beam is displaced upward $(+)$ and if the
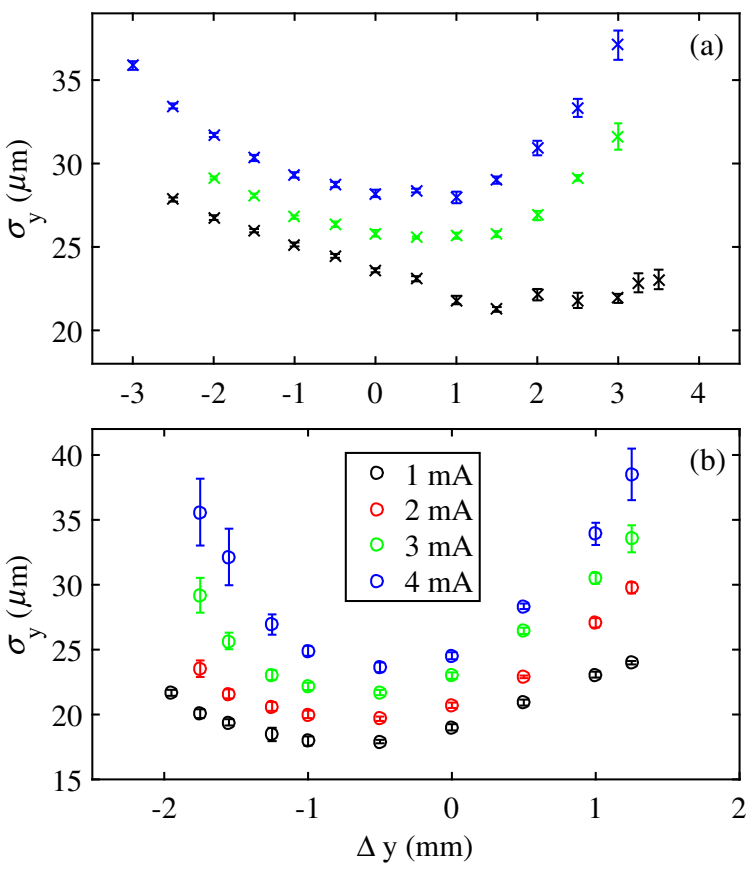

FIG. 12. The measured vertical beam size $\sigma_{y}$ as a function of the beam vertical displacement $\Delta y$ with respect to (a) the center of the scrapers and (b) the undulator chamber center at different $e^{-}$bunch currents: $1 \mathrm{~mA}$ (black), $2 \mathrm{~mA}$ (red), $3 \mathrm{~mA}$ (green), and $4 \mathrm{~mA}$ (blue). The $2 \mathrm{~mA}$ data were not taken for the scrapers.

wakefield-induced tilt has the opposite sign as the pretilt, the wakefield-induced tilt will compensate the pretilt so as to reduce the $\sigma_{y}$. Once the pretilt is totally compensated, $\sigma_{y}$ reaches the minimum. Since at the same $\Delta y$ the wakefieldinduced tilt increases with the current, $\Delta y_{\min }$ will be smaller when the bunch current is higher. The pretilt when $\Delta y=0$ could come from the dispersion at the rf cavity. It is possible that the center axis of the closed scrapers may not align with the center axis of the vacuum chamber where the scrapers were installed. This asymmetric configuration of the closed scrapers could introduce a tilt when $\Delta y=0$ but this tilt will be current dependent. The off-centered scrapers could also explain the slight asymmetry of the $\sigma_{y}$ vs $\Delta y$ curve about the $\Delta y=\Delta y_{\min }$.

To study the wakefield effect, we did similar measurements ( $\sigma_{y}$ versus $\left.\Delta y\right)$ at the narrow-gap undulator chamber. The results are shown in Fig. 12(b). Similar behavior as the scrapers was observed except that $\Delta y_{\min }$ is negative for all current data. This is understandable because the vertical phase advance between the undulator chamber and the $e^{-}$ xBSM yields a negative value of $\cos \left(\Delta \phi-\pi Q_{y}\right)$ in Eq. (5) while it is positive for the scrapers. The wakefield-induced tilt from the undulator chamber will then have the opposite sign as that from the scrapers.

As Fig. 12(b) shows, when the beam is close to the edge of the undulator chamber, the vertical beam size increase is severe, indicating the wakefield is strong at the edge. It is also likely that during measurements the beam went through the narrow-gap long chamber with a small angle, 
which may further enhance the emittance dilution. Fourth generation light sources such as MAX-IV, APS-U, and ALS-U are using small vertical aperture chambers [23-25]. More narrow-gap undulators will be installed in those accelerators. Care must be taken to ensure that the beam is on axis through the narrow-gap chambers to avoid emittance diluting effects from the wakefields.

\section{DISCUSSION}

As Fig. 8(b) shows, the $\sigma_{y}$ from tracking simulation in the lattice lat $^{+}(-54)$ with top scraper inserted increases slightly with the bunch current, which does not agree well with the measured data. This discrepancy is likely due to the nonGaussian beam profile generated by the wakefields. The measured beam size was obtained by fitting the 32 pixel data from the xBSM using a Gaussian function [20]. This fitting method catches the core part of a non-Gaussian profile. In simulation, the beam size $\sigma_{y}$ is defined as $\sqrt{\left\langle y^{2}\right\rangle}$, taking all the particles into account. Due to the non-Gaussian profile, this method tends to overestimate the vertical beam size compared to the Gaussian fitting method. Therefore, the $\sigma_{y}$ from simulation is larger than the measured data. To prove this principle, the $y$ positions of the 1000 tracking particles were binned into 32-bin histograms similar to the measured xBSM data, then fitted with a Gaussian function. The Gaussian fitting method does reduce the simulated $\sigma_{y}$ by 2-3 $\mu \mathrm{m}$ but still cannot explain the 7-8 $\mu \mathrm{m}$ difference between the simulated and the measured data. This nonGaussian beam effect is only obvious in the lattice lat ${ }^{+}(-54)$ when the wakefield-induced tilt is near zero for all currents. For other lattices, the effect is negligible.

Another possible reason for the discrepancy is that the transverse monopole wakefield obtained from the T3P simulation may not represent the actual wakefield that the beam encountered. In our T3P simulation, the scraper was modeled as a perfect conducting material. In reality, the scrapers are made of stainless steel while the surrounding vacuum chamber is aluminum. The resistive wall effect from these parts may elongate the transverse monopole wake [Fig. 5(b)] in time so as to reduce the tilt (the effective tilt factor would be less). However, a reasonable estimate shows that the transverse wakefield of the asymmetric scrapers due to the resistive wall is 3 orders of magnitude less than the geometric wake [26]. Hence, though we have not included the resistive wall contribution in T3P so far, we do not expect that doing so will significantly change the T3P wakes. It is worth noting that the bunch length increases with current due to potential well distortion $[6,27]$. The bunch lengthening may stretch the transverse monopole wake in time to reduce the tilt. However, according to Eq. (7) the $\sigma_{y}$ could remain unchanged because of reducing $\theta_{y z}$ and increasing $\sigma_{z}$ concurrently. Simulations including the bunch lengthening due to the potential well distortion will be needed.

The non-Gaussian beam profile induced by the wakefields of the asymmetric scraper configuration could be interesting to measure directly. We propose to use a streak camera to view the beam profile in the $y z$ plane (side view), a technique that has been demonstrated at CERN to measure the head-tail instability [28]. Measuring the beam halo of this non-Gaussian profile could be another interesting project since more particles could be in the tails when the wakefields are present. The asymmetric scrapers then could serve as a useful tool for adjusting the beam halo in a circular accelerator.

\section{CONCLUSION}

Current-dependent vertical beam size growth was observed with an asymmetric vertical scraper configuration (top scraper inserted, bottom scraper retracted). This vertical beam size growth was found to be due to vertical-longitudinal coupling induced by the wakefield of the asymmetric scrapers. The transverse monopole wake was calculated using $\mathrm{T} 3 \mathrm{P}$, and then incorporated into a particle tracking code. The simulated $\sigma_{y}$ agrees very well with the measurements, confirming that the $\sigma_{y}$ growth is due to the $y z$ tilt of the beam. In addition, we found that a preexisting residual $y z$ tilt could be compensated by the scraper wake.

Our observations suggest that the vertical beam size growth observed during intrabeam scattering studies in CESRTA may indeed be due to asymmetries in the beam pipe, such as pumping slots. However, additional work is needed for a quantitative test of this hypothesis.

We note that misalignment of the closed orbit through a narrow vertical aperture (a narrow-gap undulator, for example) will generate a transverse wakefield that can contribute to an increase in vertical beam size. Measurements of the projected vertical beam size as a function of vertical displacement through a narrow-gap undulator were found to be useful in identifying a preexisting $y z$ tilt.

\section{ACKNOWLEDGMENTS}

The authors thank Will Bergan for his assistance with recent machine studies; Mike Billing for many helpful discussions; Joe Calvey for developing the initial tools for tracking simulations with wakefields and for his work on the initial scraper machine studies; Mike Ehrlichman for developing the first CUBIT models and initiating the т3P wakefield calculations; Aaron Lyndaker for providing information on the undulator chambers; Dave Sagan for his assistance with BMAD; Jim Shanks for his help with low emittance tuning; and Cho $\mathrm{Ng}$ (SLAC) for his assistance with T3P. This research was supported by NSF PHYS1068662 and PHYS-1416318.

\section{APPENDIX: THE $y z$ TILT DUE TO TRANSVERSE WAKEFIELD USING ONE-TURN MATRIX}

The $y z$ tilt due to the wakefield can also be derived in terms of the one-turn matrix. In the case where the $x y$ and $x z$ coupling can be ignored and the vertical dispersion is zero, the full turn matrix has the form 


$$
\mathbf{T}=\left(\begin{array}{ccc}
\mathbf{X} & \mathbf{0} & \mathbf{0} \\
\mathbf{0} & \mathbf{M} & \mathbf{m} \\
\mathbf{0} & \mathbf{n} & \mathbf{N}
\end{array}\right)
$$

where $\mathbf{X}, \mathbf{M}, \mathbf{m}, \mathbf{n}$, and $\mathbf{N}$ are $2 \times 2$ matrices and 0 is the $2 \times 2$ matrix of zeros. In order to obtain the tilt at the observation point, we need to calculate the $\mathbf{H}$ matrix from the one-turn matrix at the observation point, which determines $\zeta_{b}$ and $\eta_{b}$ in Eq. (2):

$$
\zeta_{b}=\mathbf{H}_{11}, \quad \eta_{b}=\mathbf{H}_{12}
$$

The solution for $\mathbf{H}$ is given by [12,29]

$$
\begin{aligned}
\mathbf{H} & =\frac{\mathbf{m}+\mathbf{n}^{\dagger}}{b \chi} \\
b & =\sqrt{\frac{1}{2}+\frac{\sqrt{\operatorname{Tr}(\mathbf{M}-\mathbf{N})^{2}}}{2 \chi}} \\
\chi & =\sqrt{\operatorname{Tr}(\mathbf{M}-\mathbf{N})^{2}+4\left|\mathbf{m}+\mathbf{n}^{\dagger}\right|},
\end{aligned}
$$

where $\operatorname{Tr}(\cdot)$ is the matrix trace and $\mathbf{n}^{\dagger}$ is the symplectic conjugate matrix of $\mathbf{n}$.

Considering a scenario in which the beam size is measured at point 0 , the wake element produces a kick at point 1 , and the $\mathrm{rf}$ cavity is at point 2 , the one-turn matrix at point 0 can be written as

$$
\mathbf{T}=\mathbf{T}_{10} \mathbf{T}_{\mathrm{s}} \mathbf{T}_{01},
$$

$$
\mathbf{M}_{01}=\left(\begin{array}{cc}
\sqrt{\frac{\beta_{1}}{\beta_{0}}}\left(\cos \Delta \phi_{01}+\alpha_{0} \sin \Delta \phi_{01}\right) & \sqrt{\beta_{1} \beta_{0}} \sin \Delta \phi_{01} \\
\frac{\left(\alpha_{0}-\alpha_{1}\right) \cos \Delta \phi_{01}-\left(1-\alpha_{0} \alpha_{1}\right) \sin \Delta \phi_{01}}{\sqrt{\beta_{0} \beta_{1}}} & \sqrt{\frac{\beta_{0}}{\beta_{1}}}\left(\cos \Delta \phi_{01}-\alpha_{0} \sin \Delta \phi_{01}\right)
\end{array}\right),
$$

where $\Delta \phi_{01}$ is the vertical phase advance from 0 to $1, \alpha_{0}, \beta_{0}$ and $\alpha_{1}, \beta_{1}$ are the Twiss parameters at point 0 and point 1 , respectively, and

$$
\mathbf{N}_{01}=\left(\begin{array}{cc}
1 & L_{01} \alpha_{01}^{p} \\
0 & 1
\end{array}\right),
$$

where $L_{01}$ and $\alpha_{01}^{p}$ are the fraction of the total circumference from 0 to 1 and effective momentum compaction between 0 and 1 , respectively, and

$$
\mathbf{N}_{\mathrm{rf}}=\left(\begin{array}{cc}
1 & 0 \\
\tilde{V} & 1
\end{array}\right)
$$

where

$$
\tilde{V}=\frac{e V_{\mathrm{rf}} \omega_{\mathrm{rf}}}{c \beta_{r} E}
$$

where $\mathbf{T}_{\mathrm{s}}$ is the transfer matrix of the wake element (the vertical scraper). It has the form

$$
\mathbf{T}_{\mathrm{s}}=\left(\begin{array}{cc}
\mathbf{I} & \mathbf{m}_{\mathrm{s}} \\
\mathbf{m}_{\mathrm{s}} & \mathbf{I}
\end{array}\right)
$$

where $\mathbf{I}$ is the $2 \times 2$ unit matrix and $\mathbf{m}_{\mathrm{s}}$ is

$$
\mathbf{m}_{\mathrm{s}}=\left(\begin{array}{cc}
0 & 0 \\
W & 0
\end{array}\right),
$$

where $W$ is the vertical-longitudinal coupling term, related to the transverse wake $W_{\perp}$. If the rf cavity is between point 1 and point 0 , the transfer map $\mathbf{T}_{10}$ can be written as

$$
\mathbf{T}_{10}=\mathbf{T}_{20} \mathbf{T}_{\mathrm{rf}} \mathbf{T}_{12},
$$

where $\mathbf{T}_{\mathrm{rf}}$ is the transfer matrix of the rf cavity, written as

$$
\mathbf{T}_{\mathrm{rf}}=\left(\begin{array}{cc}
\mathbf{I} & 0 \\
0 & \mathbf{N}_{\mathrm{rf}}
\end{array}\right) .
$$

Since we assume the lattice has no vertical dispersion, the matrices $\mathbf{T}_{01}, \mathbf{T}_{12}$, and $\mathbf{T}_{20}$ have zero off-diagonal terms similar to Eq. (A10). We then calculate the one-turn matrix at point 0 as

$$
\mathbf{T}=\left(\begin{array}{cc}
\mathbf{M} & \mathbf{M}_{10} \mathbf{m}_{\mathrm{s}} \mathbf{N}_{01} \\
\mathbf{N}_{20} \mathbf{N}_{\mathrm{rf}} \mathbf{N}_{12} \mathbf{m}_{\mathrm{s}} \mathbf{M}_{01} & \mathbf{N}_{20} \mathbf{N}_{\mathrm{rf}} \mathbf{N}_{02}
\end{array}\right) .
$$

To evaluate $\mathbf{m}$ and $\mathbf{n}^{\dagger}$, we populate Eq. (A11) with

$\beta_{r}$ is the relativistic beta, $\omega_{\mathrm{rf}}$ is the $\mathrm{rf}$ frequency, and $V_{\mathrm{rf}}$ is the peak cavity voltage.

When the one-turn matrix $\mathbf{T}$ is evaluated, $\zeta_{b 0}$ and $\eta_{b 0}$ can be calculated using Eqs. (A2) and (A3). The $y z$ tilt angle is then obtained using Eq. (2):

$$
\begin{aligned}
\theta_{y z}= & \frac{W \sqrt{\beta_{0} \beta_{1}}}{b \chi}\left\{2 \sin \pi Q_{y} \cos \left(\pi Q_{y}-\Delta \phi_{01}\right)\right. \\
& \times\left(1-\frac{\alpha_{c}}{\beta_{c}} L_{01} \alpha_{01}^{p}\right)+\sin \Delta \phi_{01} \\
& \left.\times\left[L \alpha_{p}+\tilde{V} L_{12} \alpha_{12}^{p}\left(1+\frac{\alpha_{c}}{\beta_{c}} L_{20} \alpha_{20}^{p}\right)\right]\right\} .
\end{aligned}
$$

In the case of small synchrotron tune, $\chi \propto 4 \sin ^{2} \pi Q_{y}$. For CESRTA lattices, $\alpha_{c} / \beta_{c}$ is small $(\sim 0.016)$. We can see the first term in Eq. (A16) has a similar form as Eq. (5). 
[1] A. Chao, Physics of Collective Beam Instabilities in High Energy Accelerators (John Wiley and Sons, New York, 1993).

[2] Y. Chae, Advanced Photon Source, Argonne National Laboratory Technical Report No. ANL/APS/LS-318, 2009.

[3] P. Brunelle, R. Nagaoka, and R. Sreedharan, Measurement and analysis of the impact of transverse incoherent wakefields in a light source storage ring, Phys. Rev. ST Accel Beams 19, 044401 (2016).

[4] S. T. Wang, D. L. Rubin, and J. P. Shanks, CESR Lattice for Two Beam Operations with Narrow Gap Undulators at CHESS, in Proceedings of 7th International Particle Accelerator Conference (IPAC'16), Busan, Korea, 2016 (JACoW, Geneva, 2016), pp. 2968-2971.

[5] M. Palmer et al., in Proceedings of the 23rd Particle Accelerator Conference, Vancouver, Canada, 2009 (IEEE, Piscataway, NJ, 2009), pp. 4200-4204.

[6] M. P. Ehrlichman, W. Hartung, B. Heltsley, D. P. Peterson, N. Rider, D. Rubin, D. Sagan, J. Shanks, S. T. Wang, R. Campbell, and R. Holtzapple, Intrabeam scattering studies at the Cornell Electron Storage Ring Test Accelerator, Phys. Rev. ST Accel. Beams 16, 104401 (2013).

[7] J. Calvey et al., Measurement and Modeling of Single Bunch Wake Field Effects in CE, in Proceedings of 6th International Particle Accelerator Conference, Richmond, 2015 (JACoW, Geneva, 2015), pp. 681-684, http://jacow .org/IPAC2015/papers/mopma056.pdf.

[8] S. T. Wang, J. D. Perrin, S. Poprocki, and D. L. Rubin, Experimental Study of Vertical-Longitudinal Coupling Induced by Wakefields at CesrTA, in Proceedings of 8th International Particle Accelerator Conference (IPAC'17), Copenhagen, Denmark, 2017 (JACoW, Geneva, 2017), pp. 3200-3203.

[9] S. Heifets, A. Wagner, and B. Zotter, SLAC Technical Report No. SLAC-AP-110, 1998.

[10] G. Stupakov, SLAC Technical Report No. SLAC-PUB8683, 2000.

[11] A. B. Temnykh et al., Construction of CHESS compact undulator magnets at Kyma, in Proceedings of SPIE 9512, Advances in $x$-ray free-electron lasers instrumentation III, Prague, Czech Republic, 2015 (SPIE, 2015).

[12] M. P. Ehrlichman, A. Chatterjee, W. Hartung, B. Heltsley, D. P. Peterson, N. Rider, D. Rubin, D. Sagan, J. P. Shanks, and S. T. Wang, Measurement and compensation of horizontal crabbing at the Cornell Electron Storage Ring Test Accelerator, Phys. Rev. ST Accel. Beams 17, 044002 (2014).

[13] K. Ko et al., in Proceedings of the 25th International Linear Accelerator Conference, LINAC-2010, Tsukuba, Japan (KEK, Tsukuba, Japan, 2010), p. 1028.
[14] CUBIT 14.1, Sandia National Laboratories, Albuquerque, NM, cubit.sandia.gov.

[15] NERSC, Lawrence Berkeley National Laboratory, Berkeley, CA, www.nersc.gov.

[16] W. Panofsky and W. Wenzel, Some considerations concerning the transverse deflection of charged particles in radio-frequency fields, Rev. Sci. Instrum. 27, 967 (1956).

[17] D. Sagan, Bmad: A relativistic charged particle simulation library, Nucl. Instrum. Methods Phys. Res., Sect. A 558, 356 (2006).

[18] A. Wolski, Alternative approach to general coupled linear optics, Phys. Rev. ST Accel. Beams 9, 024001 (2006).

[19] J. Shanks, D. L. Rubin, and D. Sagan, Low-emittance tuning at the Cornell Electron Storage Ring Test Accelerator, Phys. Rev. ST Accel. Beams 17, 044003 (2014).

[20] J. P. Alexander et al., Vertical beam size measurement in the CESR-TA $e^{+} e^{-}$storage ring using x-rays from synchrotron radiation, Nucl. Instrum. Methods Phys. Res., Sect. A 748, 96 (2014).

[21] S. T. Wang, D. Rubin, J. Conway, M. Palmer, D. Hartill, R. Campbell, and R. Holtzapple, Visible-light beam size monitors using synchrotron radiation at CESR, Nucl. Instrum. Methods Phys. Res., Sect. A 703, 80 (2013).

[22] J. Snuverink, R. Ainsworth, S. T. Boogert, F. J. Cullinan, A. Lyapin, Y. I. Kim, K. Kubo, S. Kuroda, T. Okugi, T. Tauchi, N. Terunuma, J. Urakawa, and G. R. White, Measurements and simulations of wakefields at the Accelerator Test Facility 2, Phys. Rev. ST Accel. Beams 19, 091002 (2016).

[23] E. Wallén, in Proceedings of the International Particle Accelerator Conference, Kyoto, Japan (ICR, Kyoto, 2010), pp. 3171-3173.

[24] M. Borland, Advanced Photon Source, Argonne National Laboratory Technical Report No. ANL/APS/LS-337, 2013.

[25] C. Steier et al., Proposal for a Soft X-ray Diffraction Limited Upgrade of the ALS, in Proceedings of 5th International Particle Accelerator Conference (IPAC'14), Dresden, Germany (JACoW, Geneva, 2014), p. 567.

[26] M. Billing, Note on scraper resistive wall (unpublished).

[27] R. Holtzapple, M. Billing, D. Hartill, M. Stedinger, and B. Podobedov, Single bunch longitudinal measurements at the Cornell Electron-Positron Storage Ring, Phys. Rev. ST Accel. Beams 3, 034401 (2000).

[28] E. Rossa et al., Real time measurement of bunch instabilities in LEP in three dimensions using a streak camera, in Proceedings of the European Particle Accelerator Conference, Berlin, Germany, 1992 (World Scientific, Singapore, 1992), p. 144.

[29] D. Sagan and D. Rubin, Linear analysis of coupled lattices, Phys. Rev. ST Accel. Beams 2, 074001 (1999). 